\title{
Development of underground mine monitoring and communication system integrated ZigBee and GIS
}

\author{
Moridi Mohammad Ali ${ }^{\text {a, }}$, Kawamura Youhei ${ }^{\text {a, }}$, Sharifzadeh Mostafa ${ }^{\text {a }}$, Chanda Emmanuel Knox ${ }^{\text {b }}$, Wagner \\ Markus ${ }^{\mathrm{c}}$, Jang Hyongdoo a , Okawa Hirokazu ${ }^{\mathrm{d}}$
}

\author{
${ }^{a}$ Department of Mining Engineering and Metallurgy Engineering, Western Australian School of Mines (WASM), Curtin University, 6433, Australia \\ b School of Civil, Environment and Mining Engineering, The University of Adelaide, Adelaide, 5005, Australia \\ c School of Computer Science, The University of Adelaide, Adelaide, 5005, Australia \\ d Graduate School of Engineering and Resource Science Department of Materials-process Engineering and Applied Chemistry Environment, Akita University, \\ 010-8502, Japan
}

*Corresponding author

Locked Bag 30

Department of Mining and Metallurgy Engineering,

Western Australian School of Mines,

Curtin University, Kalgoorlie, Australia,

WA, 6433

Tel: +61890886160

Fax: +61 890886151

E-mail: mohammad.moridi@curtin.edu.au

\begin{abstract}
An automated underground mine monitoring and communication system based on the integration of new technologies is introduced to promote safety and health, operational management and cost-effectiveness. The proposed system integration considering wireless sensor network (WSN) assisted geographic information system (GIS) enables to monitor and control underground mining applications from surface office. Based on the capabilities of WSNs, ZigBee network is adapted for near realtime monitoring, ventilation system control and emergency communication in underground mine. ZigBee nodes were developed to sense environmental attributes such as temperature, humidity and gases concentration; switching ON and OFF ventilation fans; and texting emergency messages. A trigger action plan for monitored attributes above normal and threshold value limits is programmed in the surface GIS management server. It is designed to turn the auxiliary fans on remotely or automatically in orange condition and sending evacuation messages for underground miners in unsafe (red) condition. Multi-users operation and $3 \mathrm{D}$ visualizations are other successful achievements of the proposed system for the underground monitoring and communication.
\end{abstract}

Keywords: Wireless sensor networks, ZigBee, underground mine, safety, ventilation, mine monitoring

\section{Introduction}

Underground mine safety and health remain challenging issues in the mining industry. Death toll statistics in China's coal mines have gradually reduced from 5798 to 2631 between 2000 and 2009 [1] but fatality still occurs. The number of occupational mining fatalities in the United States' underground metal mines has fluctuated from 40 to 46 during the years 2001 to 2010. Most importantly, 33.8\% of the deaths have resulted from ignitions and explosions of gas or dust [2] in underground mining. In April 2014, two men were killed when a wall collapsed in an underground coal mine in New South Wales, Australia. Human errors were concluded from reports as the most significant reasons for mining fatalities. Thus, safety is always a significant concern in mining operation. Some studies have recently focused on improving the health for underground miners. Laney and Attfield have drawn attention to the fact that the prevalence of coal workers' pneumoconiosis or progressive massive fibrosis increased from 1990 to 2000 among United States underground miners [3]. Therefore, specific consideration of both safety and health issues deserves priority in mine operation management and engineering designs to provide and maintain a safe and healthy workplace. In response to these challenges, mine automation by new technologies such as wireless sensor network (WSN) assisted with geographic information system (GIS) has been widely utilised in underground mines to enhance safety and health, productivity and reduce operational costs $[4,5]$.
The underground WSNs consist of a few to several hundred nodes between a surface gateway and specified sensor nodes at underground levels [6]. ZigBee based on IEEE 802.15.4 protocol is a new wireless sensor technology which has more benefits than other WSNs for underground monitoring and communication systems [7]. Even though ZigBee technology provides only a low data rate, its benefits are low power consumption, very cost-effective nodes, network installation and maintenance [8]. It is also capable of providing networking applications for data transmission between nodes (node to node relays) with high performance based on many wireless hops. It does not require any access point or central node to transmit data between clusters. Significance of ZigBee in underground mines compared to other WSNs was evaluated in the recent publication of authors [9].

GIS is new technology used for spatial data analysis in order to capture, store, analyse, manage, and present data that is linked to locations [10]. GIS allows users to view, understand, question, interpret, and visualize data in many ways that reveal relationships, patterns, and trends in the form of maps, globes, reports, and charts. Web-GIS is an inevitable trend which helps solve the problems of spatial information integration and sharing in technical aspect of web media $[11,12]$. Recently, researchers have technically focused on the GIS supports for the management of emergency and unsafe conditions [13-15].

In this study underground safety and health concerns are significantly mitigated based on the system integration which 
incorporates ventilation management and emergency message texting. The system integration based on the development of ZigBee nodes is introduced to sense the underground mine environment, to regulate ventilation system and to communicate between surface offices and miners. Therefore, reduced power consumption, near real-time monitoring of the environment and bilateral communicating between surface and underground personnel are achieved. Experimental tests were carried out to verify network reliability and security of the packet delivery in underground mines. The architecture of underground monitoring and communication for the system integration is illustrated in Fig. 1. Temporal ZigBee data including messages and environmental attribute readings such as temperature, humidity and gases concentration are transferred to GIS management server in the surface control centre. The transmitted data is received and stored by ZigBee program then provided for manipulation in the control centre. Risk situations are immediately identified and responded through a logical process of data analysis in the GIS management server before reaching dangerous (unsafe) levels and accidents occurring. The ventilation system management is also used for the workplace health and safety compliance and the optimisation of mine site power usage.

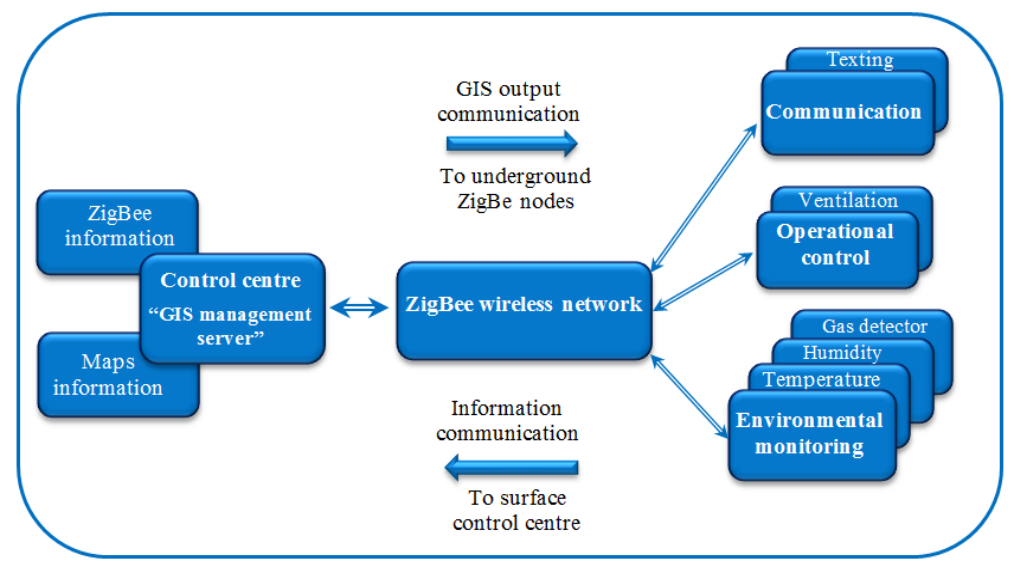

Fig. 1. Architecture of monitoring and communication system in underground mines

The remainder of this paper is organised as follows. The fundamental knowledge of ZigBee technology and GIS are first described. Then, the implementation and structure of system integration are demonstrated. Finally, the strategic process of combining ZigBee data and map information through the GIS management server is modelled for monitoring, communication and controlling the environmental attributes in an underground mine.

\section{Background of ZigBee and GIS system integration}

\subsection{ZigBee network}

ZigBee has recently been used in the field of mine safety for a range of applications mostly in underground coal mines as an automatic meter reading system, security system and remote control alongside supporting other WSNs [16,17]. The reliability and security of the ZigBee network performance have nevertheless been extensively considered. The ZigBee network applications in underground mines are limited considering the narrow bandwidth of up to $250 \mathrm{kbps}$ at a frequency of $2.4 \mathrm{GHz}$ [18]. To support the performance of these applications, ZigBee networks profit from IEEE 802.15.4 protocol [19]. IEEE 802.15.4 characterises the physical and medium access control layers of wireless personal area networks. The physical layer is the hardware for the network communication and enables operation in different frequency ranges. The medium access control layer is responsible in providing reliable data transmission and communications between a node and its immediate neighbours. It also avoids data collisions and improves network efficiency [20].
The reliability of ZigBee network under outdoor condition was verified by our team research [15], but the narrow space of underground environments has significantly intensified the signal strength to transmit data between two fixed nodes for a specified distance [21]. A recent study by authors has confirmed a similar conclusion and showed a stable communication of packet deliveries between fixed ZigBee nodes for the underground monitoring and communication system in different openings [9]. Consequently, ZigBee applications considering developed ZigBee nodes by our group research for underground monitoring and communication in the field of safety and health are investigated in real cases. Based on the experiments, to utilize underground ZigBee applications of sensing the environment and texting messages, scheduling specific times for data transmission through the network are required. In other words, measuring and sending data by sensor nodes are set in different time intervals to avoid any network congestion and to improve network performance.

\subsection{Geographic information system}

GIS is based on computer programs used for storage, modelling, retrieval, mapping and analysis of geographic data. In this system, spatial features of a specified environment are stored and manipulated in a coordinate system, which refers to a specific place. GIS merges multi-layers of required geographic and spatial data for the user evaluation, and helps determine the locations and times of possible incidents in advance. Fig. 2 illustrates a cycle of GIS to process data and layers for the purpose of risk assessment in underground mine sites. GIS server is capable of managing and processing data for a substantial number of attributes coming from different sources. It also is able to distribute and share data between 
users based on internet or intranet, and data could be saved, manipulated or informed by other users. Therefore, GIS can decrease the time and cost of sharing geographic data and its attributes.

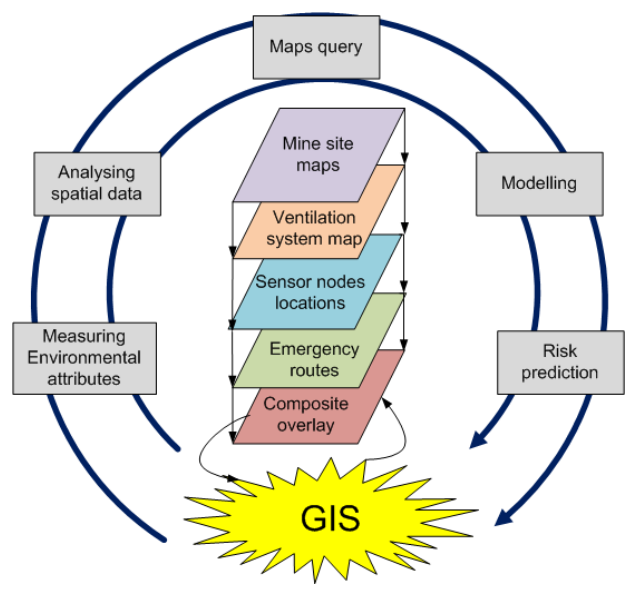

Fig. 2. GIS data process cycle and geographic layers in an underground mine

\subsection{System integration}

In the challenging environment and changing topology of a mine, reliable and simplified communication is a high-stake issue with the objectives of safe and efficient mining operations. Automation of remote and automatic systems has improved workplace safety and health for miners, yielded cost-effectiveness, management improvement of technical problems, energy savings, real-time response to incidents. In response to these challenges, integration of technologies has a significant role in underground mining automation. According to WSNs' specific features of high reliability and multi-hop networking, ZigBee can create an integrated wireless network between nodes in the underground mine tunnels and the surface gateway. In this study, ZigBee's capability of monitoring underground environmental attributes is combined with geographic information to provide potential applications in communication, operational and environmental monitoring systems of underground mining.

In order to achieve such smart underground mine system, integrating maps information and spatio-temporal data from ZigBee nodes into a database at a control centre is required. Fig. 3 illustrates data processing and results management in the surface control centre.

The network demanded in an underground mine must be capable of providing bilateral communications between the surface control centre and all underground wireless nodes interactively. According to the threshold limit values for the different variable parameters $\left(\mathrm{V}_{1}, \mathrm{~V}_{2}, \ldots, \mathrm{V}_{\mathrm{n}}\right)$ of underground mine environment, the conditions of safe, transient and unsafe were set. Thus, the remote or automatic countermeasures in a GIS management server were arranged in order to control ventilation fans and send alert or alarm messages to relevant authorities. Additionally, immediate texting messages are bilaterally communicated between underground personnel and the surface operator in emergency conditions.

Based on this system, near real-time monitoring data, remote and automatic controls, and communication by texting messages have achieved the required safety and health outcomes and improving underground mining operations. Such achievements are more efficient for emergency management when system configuration enables control, monitoring and communication between users in various places connected by internet medium access.

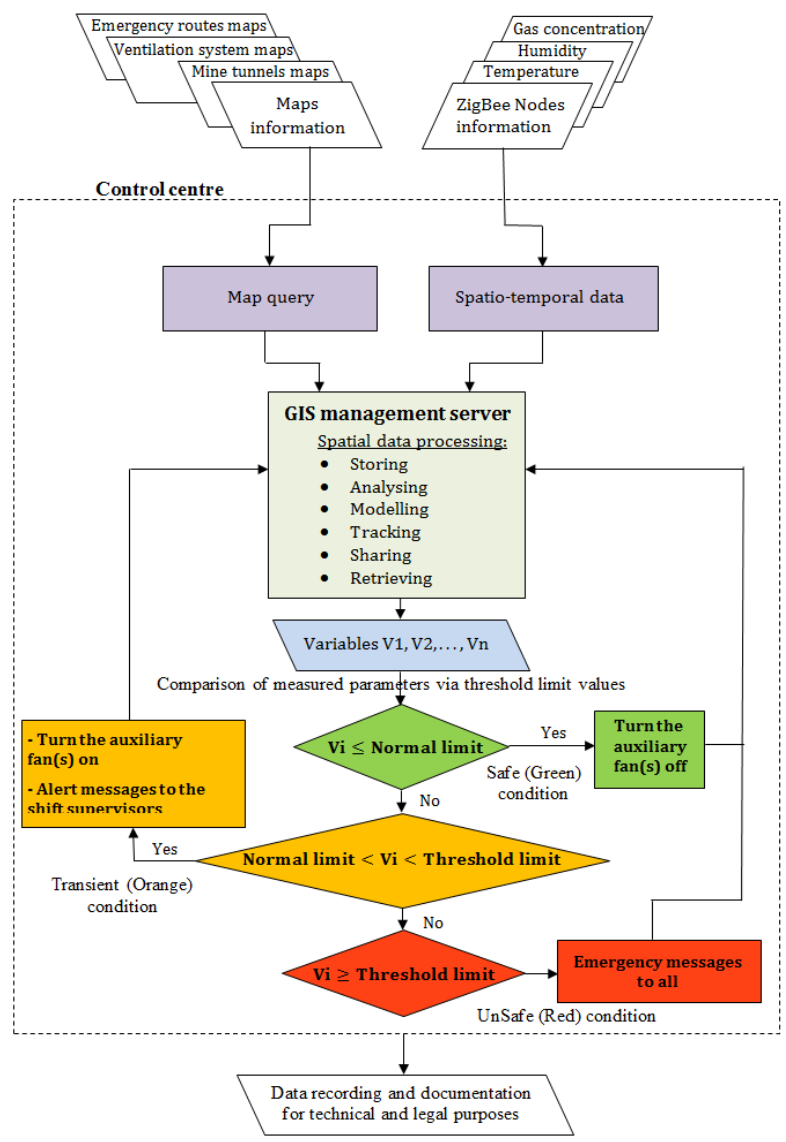

Fig. 3. Flow chart of data processing and result management 


\section{System structure}

\subsection{Wireless network setup}

The entire system of the tested underground WSN is composed of different ZigBee nodes such as coordinator, routers and end devices. These products were developed in collaboration with Tokyo Cosmos Electric Co., Ltd. The JN5148-EK010 kit (Jennic) stacks were employed to create ZigBee network. The wireless network initially is created by coordinator (gateway) to join other nodes. A ZigBee coordinator connected to laptop (PC) using in the experiments is illustrated in Fig. 4. Bilateral communication was provided between the coordinator and end devices to send and receive messages and readings instantaneously taken by their sensors. Routers with the ability of sensing the environment were employed to relay communication through the network. In addition, sending and receiving messages and remote control of ventilation fans are enabled by the surface coordinator based on the designed software (Fig. 4).

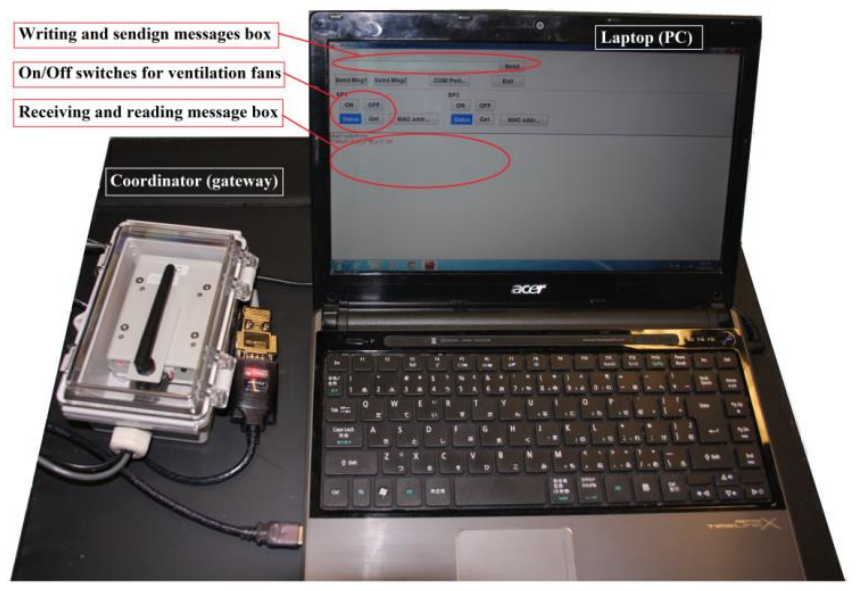

Fig. 4. ZigBee coordinator connected to laptop (PC)

To setup WSNs, power consumption and high reliability of packet delivery are the most concerns. For the former case, ZigBee nodes are configured to transmit data in longer periods when the mine is in safe and transient conditions which it is caused to extend the life of batteries. In latter case, different time intervals are considered for data delivery of environment sensing to avoid network congestion and possibility of packets loss. The power usage of direct and alternating currents (DC/AC) for the ZigBee nodes (except the coordinator) were designed to operate under battery and mine site power supply, respectively. Thus, alternating currents power usage is resulted in the extension of battery life, and ZigBee nodes are enabled to continue long-time data telemetry during power outages at any accident. The ZigBee nodes can last a few days to several months depends on their data rate and applications.

\subsection{Sensing environment}

The safety and health of coal and metal/non-metal mining operations were raised considerably as the result of the wireless monitoring of environment attributes. Digital temperature-humidity compound sensor on-board of each JN5148 with advanced sensitivity and long-term stability for mine sites is utilised in the system. Methane, Oxygen, $\mathrm{CO}_{2}$,
$\mathrm{CO}, \mathrm{NO}_{\mathrm{X}}$ and $\mathrm{SO}_{2}$ concentration sensors (readers) are easily connected to ZigBee nodes to sense the environment. The sensors were configured the single-line communication to transmit real-time data to the nodes. The measurement of $\mathrm{CO}_{2}$ concentration in this study was considered to manage safety and health risks nearby coal strata in coal mines or fumesfilled spaces in metal/non-metal mines.

\subsection{Text messaging operators}

Developed ZigBee nodes are enabled to connect with laptops and mobile phones for sending and receiving text messages. Fig. 5 illustrates portable radio stations to connect laptop (Tablet) which are designed to be placed in an underground refuge chamber and mobile phones for emergency purposes. The radio station is getting a significant role for wireless communication with surface operator during accidents particularly when cable damage or power outage occurs. Even though, its primary role is the remote control of ventilation fans. ZigBee nodes were placed in the boxes to minimise environmental effects on their operation.

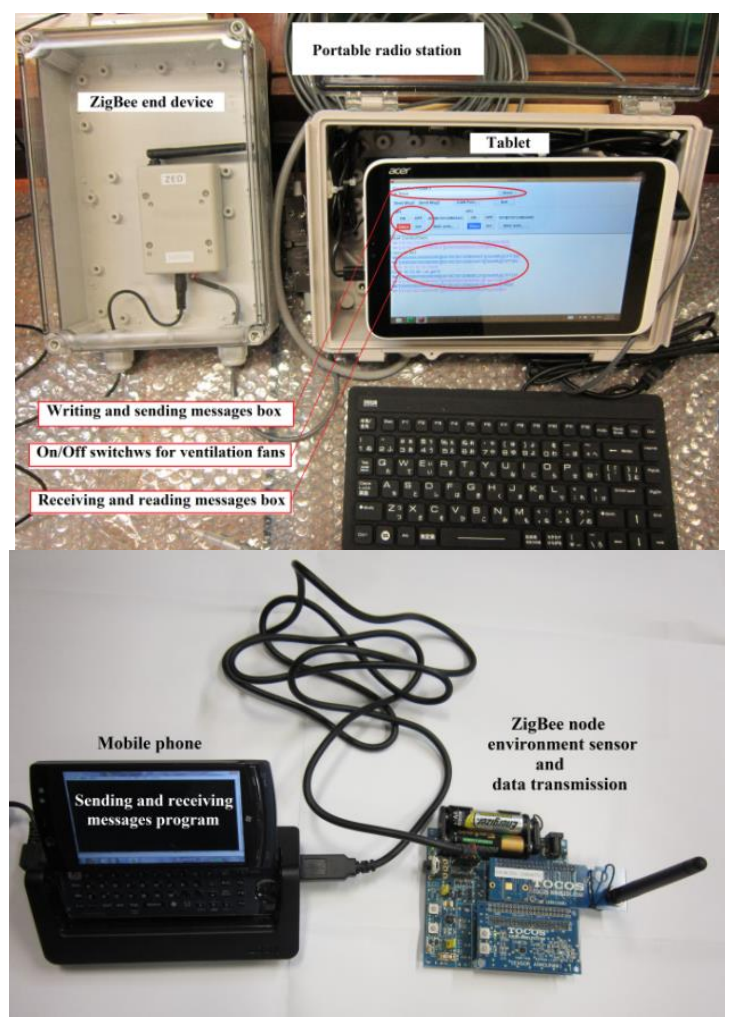

Fig. 5. Portable ZigBee radio stations to communicate between laptop and mobile phones

\subsection{Ventilation control}

Air ventilation deficiency in underground mines is a critical issue to the occupational safety and health of mine personnel. Moreover, optimization of the fans power consumption to supply underground fresh air is considered on ventilation system design. Therefore, adding auxiliary fans to the ventilation system is economically required to improve air quality during hot seasons, blasting, any gas leakages and increase of exhaust fumes. In the proposed system, remote and automatic controls of auxiliary fans were programmed with the software installed on PCs locate at the surface office and refuge chamber. Fig. 6 illustrates ZigBee node with the 
ability of wireless connection to the (auxiliary) fans. Special computer interface with ON/OFF switches and receiving/sending messages installed on PCs (laptop and tablet) is illustrated on Fig. 7. In the computer interface, separate command icons were designed for each auxiliary fan which gives the user the ability of the ventilation system control.

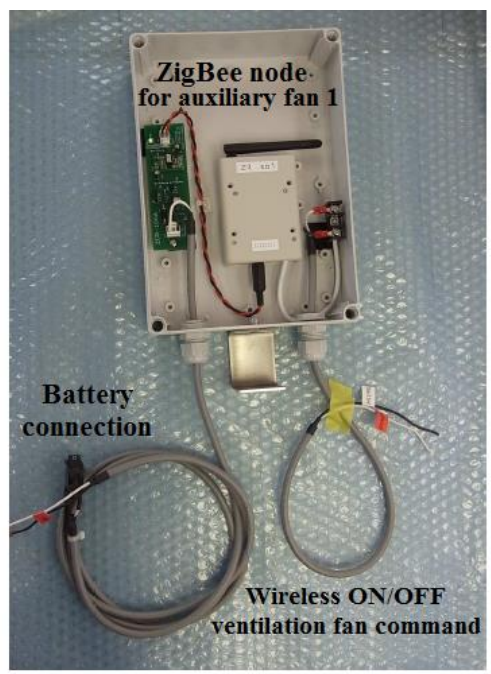

Fig. 6. ZigBee node with the ability of wireless connection to the (auxiliary) fans

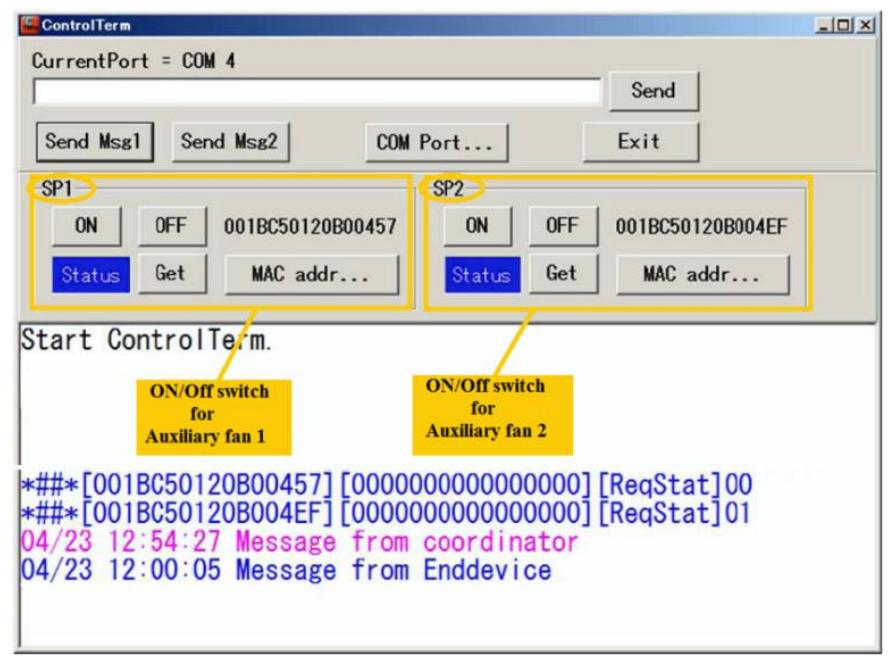

Fig. 7. Designed computer interface to switch ON/OFF the (auxiliary) fans and receiving/sending messages

\section{Data management server using GIS}

The prototype model developed in this study relies on ZigBee data and geoprocessing data of GIS. Data management server was developed on ESRI's established ArcMap 3D software, part of the ArcGIS software package.

\subsection{Input data}

The first step of our designed management server is to communicate with the outside world to receive required information. Fig. 8 illustrates data flow sheet and the variety of input data for the GIS management server. Input datasets in the database are comprised of map information, ZigBee nodes data, ZigBee text messages, ZigBee node positions, threshold limit values and contact details. Map query is primary process of map information to merge and display required features in GIS server to represent the fundamental layers of underground tunnels, geographically. These layers are revised according to the progress of underground mining activities. Then, other input data is analysed and located on the layers for further manipulation.

The quality of input dataset is considered to process and analyse at any particular database. Consequently, the quality of input data in our designed GIS management server is divided between long-term and short-term datasets. Maps, ZigBee node positions, threshold limit values and contact details are determined to be long-term input data into the database which may be periodically updated. These data are stored in attribute tables that are associated with ArcGIS geoprocessing models. ZigBee node data which measured environmental properties of mine tunnels is derived as shortterm (temporal) data. In this case, the datasets of environmental phenomena such as temperature, humidity and gas concentrations change from time to time or remain relatively continuous. Therefore, spatio-temporal data models, which show both spatial and temporal characteristics of environment, are considered as input data in the GIS management server. The spatio-temporal data is stored and manipulated in the ArcGIS geo-processing based on the related or joined table command to digital tables of data collection by ZigBee gateway software.

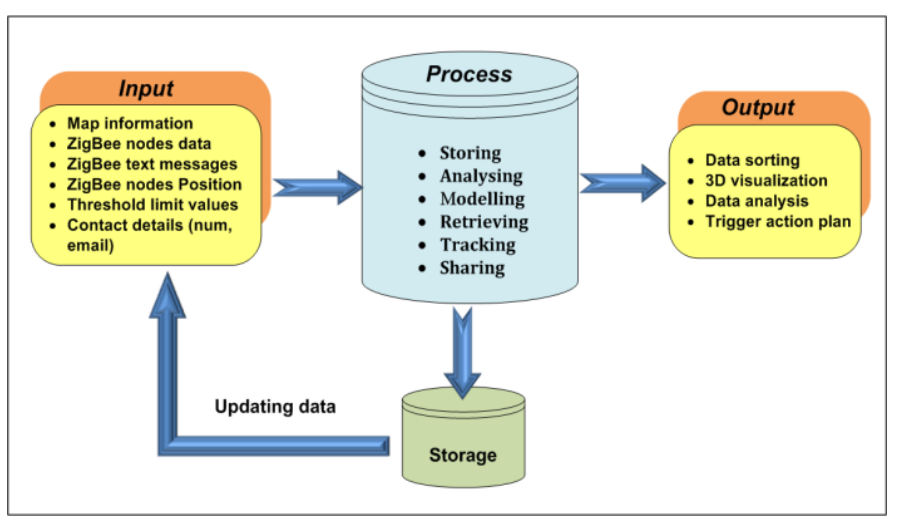

Fig. 8. Data flow sheet of integrated system in GIS server

\subsection{Process strategy}

Real-time process strategy for safe working environments involves the combination of data models and programs in GIS management server to monitor and communicate underground mine automatically and remotely. A pattern of decision making in managing spatio-temporal data was modelled as a procedure to monitor the environment attributes of underground mine tunnels (Fig. 3). To this end, near real-time and flexible scheduling strategy was planned to apply the performance of ZigBee network in an emergency status. An experiment was simulated on real maps of underground mine with developed ZigBee nodes to control ventilation fans (ON/OFF) and text emergency messages from surface control office. A section view of an underground mine and ZigBee node positions in ArcGIS screen are illustrated in Fig. 9. In this model a gateway was located in the surface control office to receive and transmit data through the underground network. The network is extended by ZigBee routers between the surface gateway and underground end devices based on optimised communication ranges. ZigBee End devices were 
divided to three groups in this experiment. One is connected to the auxiliary fans to switch them on or off automatically or remotely. Another is attached to a radio station which enables to write and read messages. The radio station can be portable or located in underground refuge room. Lastly, sensor nodes are mounted in working area which sense environment attributes such as temperature, humidity and gas concentration.

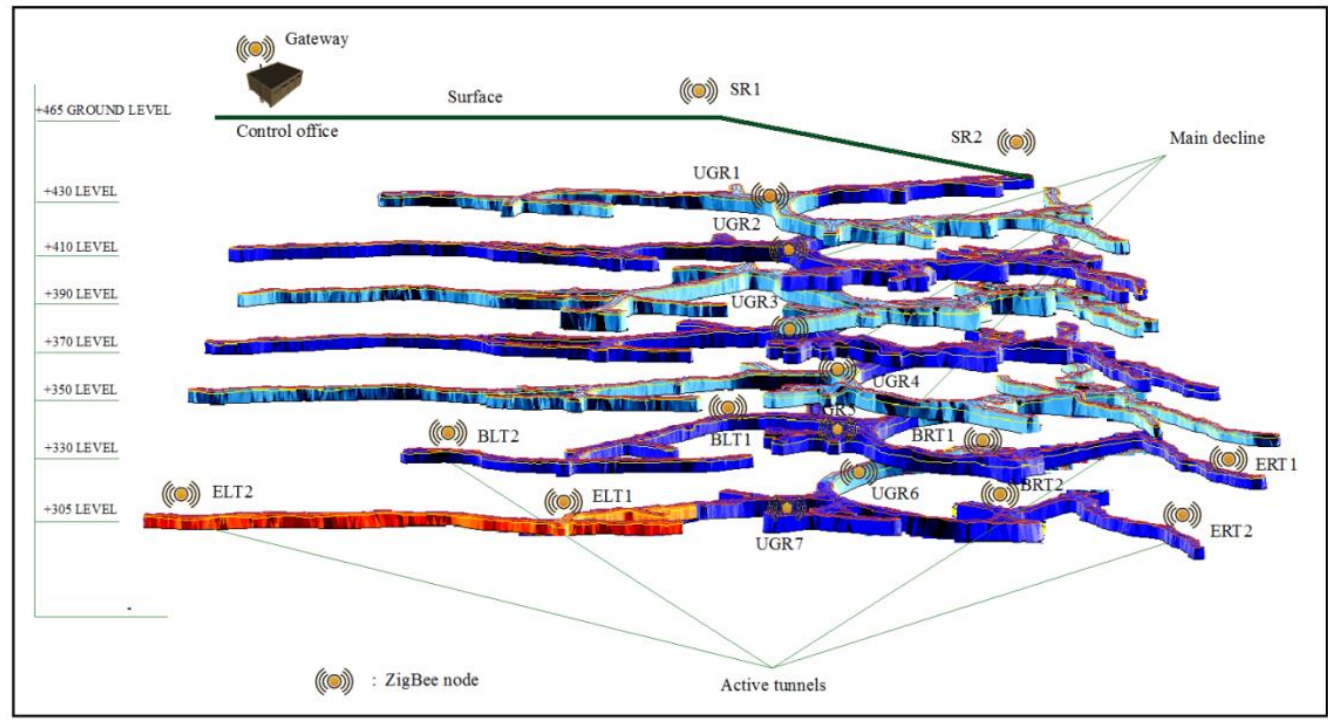

Fig. 9. A thematic map of an underground mine and ZigBee node positions in ArcGIS

The transmitted data firstly is stored in the GIS management server located at control centre. The ability of the map visualisation on GIS (ArcGIS) allows the position and component of the attributes in underground mine environment to be visually displayed on the screen. Then, the spatiotemporal data tables stored by ZigBee software in the database were joined or related to the attribute tables of node geographic positions in the geo-processing services of GIS management server. A joining table of spatio-temporal data and geographic node position created in ArcGIS (ArcMap) is illustrated in Table 1. In other words, in this joining table each node position is connected to the related and measured variable parameters including temperature, humidity and gas concentration.

\section{Table 1}

Storage of transmitted data by ZigBee gateway

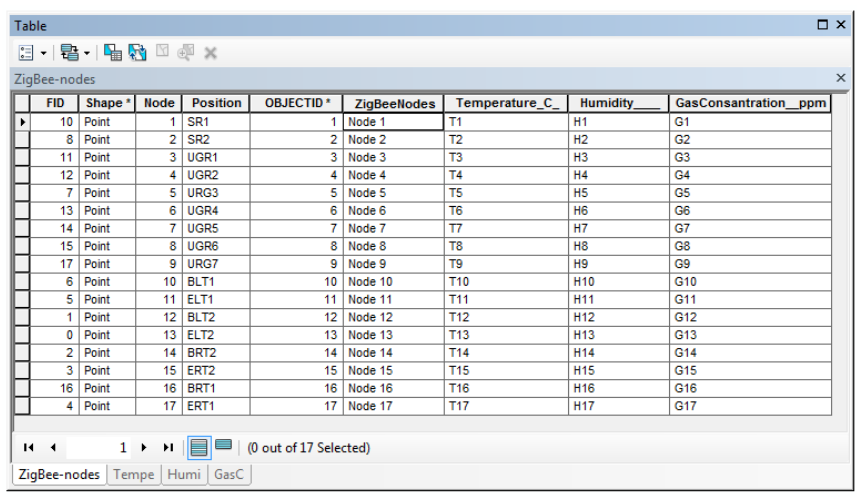

Following this, the spatio-temporal data was analysed, modelled and retrieved in the GIS management server. A geoprocessing model based on Python (ArcPy) was designed to track and control the environmental attributes in different conditions. Normal and threshold limit values to assess environmental attributes according to underground mining standards were then derived. Normal and threshold limit values for the discrete conditions of safe and unsafe statues are presented in Table 2. According to the normal and threshold limit values, the status of working environment in underground mine were assessed in three conditions of safe (green), transient (orange) and unsafe (red).

Table 2

Threshold limit values for working environments in underground mine

\begin{tabular}{|c|c|c|c|}
\hline \multirow[b]{2}{*}{ Variables $\left(\mathbf{V}_{\mathbf{i}}\right)$} & \multicolumn{3}{|c|}{ Event procedure conditions } \\
\hline & $\begin{array}{c}\text { Safe } \\
\text { (Green) }\end{array}$ & $\begin{array}{l}\text { Transient } \\
\text { (Orange) }\end{array}$ & $\begin{array}{l}\text { Unsafe } \\
\text { (Red) }\end{array}$ \\
\hline $\begin{array}{l}\text { Temperature }\left(\mathrm{T}_{1}, \mathrm{~T}_{2}, \ldots,\right. \\
\left.\mathrm{T}_{\mathrm{n}}\right),{ }^{C \textrm{C}}\end{array}$ & $\mathrm{Ti} \leq 28$ & $28<\mathrm{Ti}<40$ & $\mathrm{Ti} \geq 40$ \\
\hline $\begin{array}{l}\text { Humidity }\left(\mathrm{H}_{1}, \mathrm{H}_{2}, \ldots, \mathrm{H}_{\mathrm{n}}\right) \text {, } \\
\text { of }\end{array}$ & $\mathrm{Hi} \leq 75$ & $75<\mathrm{Hi}<85$ & $\mathrm{Hi} \geq 85$ \\
\hline $\begin{array}{l}\text { Gas concentration for } \mathrm{Co}_{2} \\
\left(G_{1}, G_{2}, \ldots, G_{n}\right), \text { ppm }\end{array}$ & $\mathrm{Gi} \leq 2000$ & $2000<\mathrm{Gi}<5000$ & $\begin{array}{c}\mathrm{Gi} \\
\geq 5000\end{array}$ \\
\hline
\end{tabular}

Finally, a loop of conditional procedures and trigger actions were set. The measured parameters (spatio-temporal data) were stored while these data are less than or equal normal limit values (safe condition). The loop was periodically retrieved each 30 minutes in order to consume less power and to extend the battery life of ZigBee nodes and reduce congestion through the network. Otherwise, a trigger plan was set for the values mounted in the range of between normal and threshold limits (transient condition) or greater than threshold limit (unsafe condition). The trigger action plan applied in the GIS management server to respond the deviation of values from normality is presented in Table 3 . In the transient (orange) condition, the auxiliary fans which had designed for emergency ventilation system would be automatically or remotely turned on. In this state, the model was also setup to send alert messages to shift supervisors. The periodic time of data reading in orange state is reduced to 15 min to ensure the safe and health conditions of underground environment in the shorter time possible. Emergency (alarm) messages in the event of unsafe (red) condition would be texted to surface authorise and to underground personnel for immediate 
evacuee from the hazardous places. The cycle time of data acquisition is minimised to 5 minutes in this situation.

Table 3

Trigger action response plan

\begin{tabular}{|c|c|c|c|}
\hline \multirow[b]{2}{*}{ Variables $\left(\mathbf{V}_{i}\right)$} & \multicolumn{3}{|c|}{ Counter measure implements } \\
\hline & $\begin{array}{c}\text { Safe } \\
\text { (Green) }\end{array}$ & $\begin{array}{c}\text { Transient } \\
\text { (Orange) }\end{array}$ & $\begin{array}{c}\text { Unsafe } \\
\text { (Red) }\end{array}$ \\
\hline $\begin{array}{l}\text { Reading time } \\
\text { interval (min) }\end{array}$ & 30 & 15 & 5 \\
\hline $\begin{array}{l}\text { Temperature }\left(T_{1} \text {, }\right. \\
\left.T_{2}, \ldots, T_{n}\right)\end{array}$ & \multirow{3}{*}{$\begin{array}{ll}\text { - } & \text { Next } \\
& \text { reading }\end{array}$} & \multirow{3}{*}{$\begin{array}{l}\text { - Turn the auxiliary } \\
\text { fan(s) on } \\
\text { - Text message to } \\
\text { the shift } \\
\text { supervisors } \\
\text { - Next reading }\end{array}$} & \multirow{3}{*}{$\begin{array}{l}\text { - } \text { Text } \\
\text { message to } \\
\text { all to } \\
\text { evacuate } \\
\text { from } \\
\text { unsafe } \\
\text { place(s) } \\
\text { - } \begin{array}{l}\text { Next } \\
\text { reading }\end{array}\end{array}$} \\
\hline $\begin{array}{l}\text { Humidity }\left(\mathrm{H}_{1}, \mathrm{H}_{2},\right. \\
\left.\ldots, \mathrm{H}_{\mathrm{n}}\right)\end{array}$ & & & \\
\hline $\begin{array}{l}\text { Gas concentration } \\
\left(\mathrm{G}_{1}, \mathrm{G}_{2}, \ldots, \mathrm{G}_{\mathrm{n}}\right)\end{array}$ & & & \\
\hline
\end{tabular}

\subsection{Outputs}

Mine safety and health were improved by intelligent maps supporting spatio-temporal data and coordinate of ZigBee nodes in this experiment. The schematic representation of integrated system outputs for underground monitoring and communication is illustrated in Fig. 10. The final outputs of GIS management server are comprised of 3D visualization monitoring of underground mine tunnels and messages texting for alert and alarm conditions. The web-GIS is another application supporting the GIS management server to promote the underground monitoring and communication system.

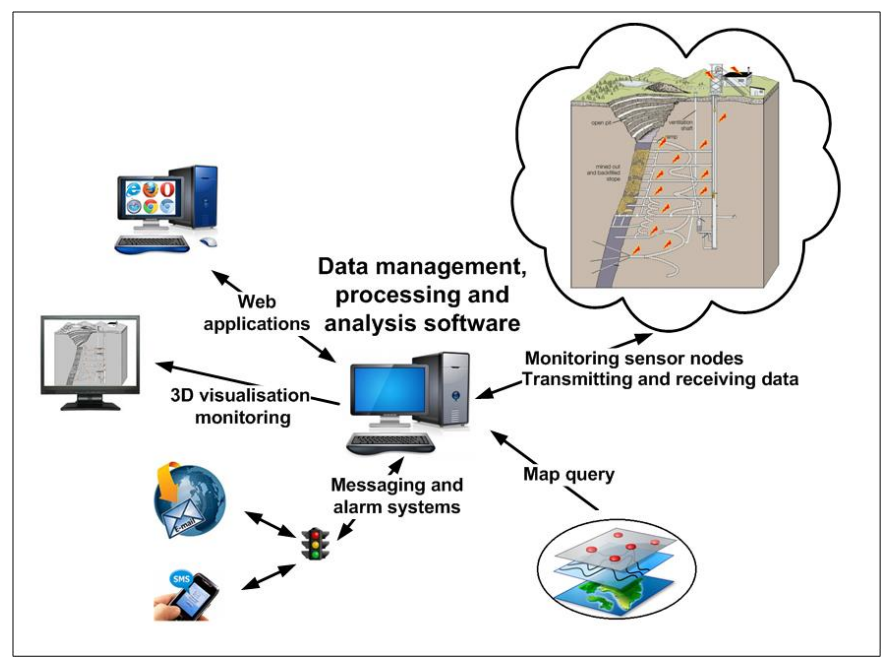

Fig. 10. Schematic representation of integrated system outputs for underground monitoring and communication

\subsection{Data storage}

Data storage and management in the central data repository of server is an essential part of the integrated system. In fact, all geographic and spatial data are stored and managed in ArcMap's geodatabase which accesses to the database at any time over the long-term. In the geodatabase, organisational structure for storing datasets and creating relationships between datasets were also provided for further analysis and interpretation. In addition, a multi-user access is enabled to work and command orders from different mine site offices.
An integrated data management and documentation to generate geospatial metadata was another approach of geodatabase automation. Metadata can create geospatial data document to investigate any genuine or non-genuine claims.

\section{Conclusion}

An integrated system based on the WSNs and GIS was introduced to automate underground mine monitoring and communication. The proposed system enhances safety and health, operational management and reduces capital costs. Considering the capability of ZigBee network and ArcGIS, the applications of real-time underground monitoring (temperature, humidity and gas concentration), ventilation system control and communication in emergency conditions by surface user would be achievable. The system is equipped with automatic or remote triggers action plans for measured environmental attributes. The measured data were classified to three categorises consisting of normal (green), transient (orange) and unsafe (red) conditions based on their values compared to normal and threshold limit values. At normal (green) condition, the measured attributes are below the normal value limits. The mining operation is continuing as it was and readings are recorded with 30 minute intervals. At the transient (orange) condition, the measurements are between normal and threshold value limits. In this state, trigger actions are become automatically active to switch the auxiliary fan on and texting message to shift supervisors. In addition, reading's intervals are reduced to 15 minutes in this situation. At unsafe (red) condition, the measurements are getting greater than threshold value limits and the system texts messages to all underground personnel for immediate evacuee from the hazardous places. Reading's intervals are reduced to 5 minutes. Furthermore, the system provides multi-users surface operation and 3D visualization for realistic understanding of underground environment and miners' conditions, and it could be a useful approach for high-tech underground mining.

\section{References}

[1] Wu L, Jiang Z, Cheng W, Zuo X, Lv D, Yao Y. Major accident analysis and prevention of coal mines in China from the year of 1949 to 2009. International Journal of Mining Science and Technology 2011; 21(5): 693-699.

[2] CDC. Centres for Disease Control and Prevention Mining Fatalities, Underground, USA, 2010.

[3] Laney AS, Attfield, MD. Coal workers' pneumoconiosis and progressive massive fibrosis are increasingly more prevalent among workers in small underground coal mines in the United States. Occupational and Environmental Medicine 2010; 67(6): 428-431.

[4] Chehri A, Fortier P, Tardif PM. UWB-based sensor networks for localization in mining environments. Ad Hoc Networks (ELSEVIER) 2009; 7(5): 987-1000.

[5] Bhattacharjee S, Roy P, Ghosh S, Misra S, Obaidat MS. Wireless sensor network-based fire detection, alarming, monitoring and prevention system for Bord-and-Pillar coal mines. Journal of Systems and Software 2012; 85(3): 571-581.

[6] Karl H, Willig A. Protocols and Architectures for Wireless Sensor Networks. England: John Wiley and Sons, Ltd; 2005. 
[7] Chen, S, Yao J, Wu Y. Analysis of the Power Consumption for Wireless Sensor Network Node Based on Zigbee. Procedia Engineering 2012; 29(0): 19941998.

[8] Shu-guang MA. Construction of Wireless Fire Alarm System Based on ZigBee Technology. Procedia Engineering 2011; 11(0): 308-313.

[9] Moridi MA, Kawamura Y, Sharifzadeh M, Chanda EK, Jang $H$. An investigation of underground monitoring and communication system based on radio waves attenuation using ZigBee. Tunnelling and Underground Space Technology 2014; 43(0): 362-369.

[10] ESRI. ArcGIS® for Emergency Management. USA; 2012.

[11] Huang $X$, Zhu W, Lu D. Underground miners localization system based on ZigBee and WebGIS. 18th International Conference on Geo-informatics; 2010.

[12] Ghorbani M, Sharifzadeh M, Yasrobi S, Daiyan M. Geotechnical, structural and geodetic measurements for conventional tunnelling hazards in urban areas - The case of Niayesh road tunnel project. Tunnelling and Underground Space Technology 2012; 31(0): 1-8.

[13] Sharifzadeh M, Mitani Y, Esaki T. Rock Joint Surfaces Measurement and Analysis of Aperture Distribution under Different Normal and Shear Loading using GIS. Rock Mechanics and Rock Engineering 2008; 41(2): 299-323.

[14] Şalap S, Karslıoğlu MO, Demirel N. Development of a GIS-based monitoring and management system for underground coal mining safety. International Journal of Coal Geology 2009; 80(2): 105-112.

[15] Kawamura Y, Dewan AM, Veenendaal B, Hayashi M, Kitahara I, Nobuhara H, Ishii K. Using GIS to Develop a Mobile Communications Network for Disaster-damaged Areas. Intrenational Journal of Digital Earth 2013; 7(4):279-293.

[16] Hongjiang H, Shuangyou W. The Application of ARM and ZigBee Technology Wireless Networks in Monitoring Mine Safety System. Paper presented at the ISECS International Colloquium on Computing, Communication, Control, and Management, CCCM; 2008.

[17] Chehri A, Farjow W, Mouftah HT, Fernando X. Design of wireless sensor network for mine safety monitoring. 24th Canadian Conference on Electrical and Computer Engineering (CCECE); 2011.

[18] Pandit V, Rane U. Coal Mine Monitoring Using ARM7 and ZigBee. International Journal of Emerging Technology and Advanced Engineering 2013; 3(5): 352359.

[19] Kim H, Chung JM, Kim CH. Secured communication protocol for internetworking ZigBee cluster networks. Computer Communications 2009; 32(13-14): 15311540 .

[20] Terada, M., 2009. Application of ZigBee sensor network to data acquisition and monitoring. MEASUREMENT SCIENCE REVIEW, 9(6), 183-187.

[21] Chehri A, Mouftah H, Fortier P, Aniss H. Experimental Testing of IEEE801.15.4/ZigBee Sensor Networks in Confined Area. The Eighth Annual Conference on Communication Networks and Services Research Conference (CNSR); 2010. 